\title{
Comparative Clinical and Economic Outcomes Associated with Warfarin Versus Apixaban in the Treatment of Patients with Venous Thromboembolism in a Large U.S. Commercial Claims Database
}

\author{
Jennifer D. Guo, PhD; Jigar Rajpura, PhD; Patrick Hlavacek, MPH; Allison Keshishian, MPH; \\ Janvi Sah, MS; Rachel Delinger, MPH; Qiao Mu, MPH; Jack Mardekian, PhD; Cristina Russ, MD; \\ Gary J. Okano, PhD; and Lisa Rosenblatt, MD
}

\begin{abstract}
BACKGROUND: Venous thromboembolism (VTE), constituting deep vein thrombosis (DVT) and pulmonary embolism (PE), is a common cause of vascular-related morbidity and mortality, resulting in a significant clinical and economic burden in the United States each year. Clinical guidelines recommend that patients with DVT and PE without cancer should be initiated on anticoagulation therapy with a direct oral anticoagulant over a vitamin $\mathrm{K}$ antagonist. Yet there is limited real-world evidence comparing the economic burden of warfarin and apixaban in treating VTE patients in a large commercially insured population.
\end{abstract}

OBJECTIVE: To compare safety and effectiveness of warfarin and apixaban and evaluate associated economic burden in treating VTE patients in a large U.S. commercial health care claims database.

METHODS: The PharMetrics Plus database was used to identify oral anticoagulant $(0 A C)$-naive patients aged $\geq 18$ years who initiated apixaban or warfarin within 30 days of a qualifying VTE encounter and had continuous health plan enrollment with medical and pharmacy benefits for 6 months before treatment initiation. Apixaban initiators and warfarin initiators were matched using the propensity score matching (PSM) technique. Cox proportional hazard models were used to assess and compare the risk of major bleeding (MB), clinically relevant nonmajor (CRNM) bleeding, and recurrent VTE. Generalized linear models were used to assess and compare the all-cause health care costs. A 2-part model with bootstrapping was used to evaluate MB- and recurrent VTE-related medical costs.

RESULTS: Among 25,193 prematched patients, 13,421 (53.3\%) were prescribed warfarin and 11,772 (46.7\%) were prescribed apixaban. After 1:1 PSM, 8,858 matched warfarin-apixaban pairs were selected with a mean follow-up of 109 days and 103 days, respectively. Warfarin was associated with a significantly higher risk of $\mathrm{MB}(\mathrm{HR}=1.52,95 \% \mathrm{Cl}=1.14-2.04)$, CRNM bleeding $(\mathrm{HR}=1.27,95 \% \mathrm{Cl}=1017.15-1.40)$, and recurrent $\mathrm{VTE}(\mathrm{HR}=1.50,95 \%$ $\mathrm{Cl}=1.24-1.82)$ compared with apixaban. Warfarin patients had significantly higher all-cause medical costs per patient per month (PPPM; \$2,333 vs. $\$ 1,992 ; P=0.001$ ), MB-related costs PPPM ( $\$ 112$ vs. $\$ 65 ; P=0.020$ ), and recurrent VTE-related costs PPPM ( $\$ 287$ vs. $\$ 206 ; P=0.014$ ) compared with apixaban patients. Warfarin patients had similar all-cause total health care costs PPPM ( $\$ 2,630$ vs. $\$ 2,420 ; P=0.051)$ compared with apixaban patients.

CONCLUSIONS: Warfarin use was associated with a higher risk of MB, CRNM bleeding, and recurrent VTE compared with apixaban. Warfarin use was also associated with higher all-cause medical costs, MB-related medical costs, and recurrent VTE-related costs PPPM compared with apixaban.

J Manag Care Spec Pharm. 2020;26(8):1017-26

Copyright $\odot 2020$, Academy of Managed Care Pharmacy. All rights reserved.

\section{What is already known about this subject}

Venous thromboembolism (VTE) is the third most common cause of vascular-related death, and the overall economic burden of VTE ranges between $\$ 13.5$ and $\$ 27.2$ billion per year in the United States.

According to the American College of Chest Physician guidelines, VTE patients who do not have cancer should be initiated on anticoagulation therapy with a direct oral anticoagulant over a vitamin $\mathrm{K}$ antagonist.

Supportive real-world evidence regarding the economic benefits of warfarin versus apixaban use with corresponding clinical outcomes in a commercially insured population is lacking.

\section{What this study adds}

This study evaluated the clinical and economic burdens of VTE treatment in warfarin initiators compared with apixaban initiators in a commercially insured population.

Warfarin initiators were associated with an approximately 1.5 times higher risk of major bleeding (MB), clinically relevant nonmajor (CRNM) bleeding, and recurrent VTE.

Warfarin initiators also had $\$ 341$ per patient per month (PPPM) higher all-cause medical costs, \$47 PPPM higher MB-related medical costs, and \$82 PPPM higher recurrent VTE-related medical costs compared with apixaban initiators.

V Tenous thromboembolism (VTE) can be classified into deep vein thrombosis (DVT) and pulmonary embolism (PE), with approximately one third of affected patients presenting with PE and two thirds with DVT. ${ }^{1}$ VTE is the third most common cause of vascular-related death, following myocardial infarction (MI) and stroke. ${ }^{2}$ Patients with comorbid conditions such as extremity trauma, cancer, surgery, prolonged immobilization, and hormone therapy are at a greater risk for VTE. ${ }^{3-8}$ These comorbidities are also strongly related to VTE recurrence, resulting in higher inpatient, outpatient, and pharmacy costs compared with those without recurrent VTE among commercially insured VTE patients. ${ }^{9}$ Incident cases of VTE along with subsequent complications reportedly result 
in an average incremental direct medical cost of $\$ 18,000$ to $\$ 23,000$ per incident case among survivors. ${ }^{10}$ Overall, the economic burden of VTE ranges between $\$ 13.5$ and $\$ 27.2$ billion per year (in 2011 U.S. dollars) in the United States. ${ }^{11}$

According to the American College of Chest Physician guidelines, DVT and PE patients without evidence of cancer should be initiated on anticoagulation therapy with a direct oral anticoagulant (DOAC: dabigatran, rivaroxaban, apixaban, or edoxaban) over a vitamin $\mathrm{K}$ antagonist (VKA) such as warfarin. ${ }^{12}$ This recommendation is supported with evidence from the AMPLIFY randomized clinical trial program that demonstrated that a 5-mg twice-daily fixed-dose oral regimen of apixaban alone (after administering apixaban $10 \mathrm{mg}$ twice daily for 7 days) was noninferior to conventional therapy (subcutaneous enoxaparin, overlapped and followed by warfarin) and was associated with significantly less bleeding in VTE patients. ${ }^{13}$

Similar to other DOACs, treatment with apixaban reduces the complexity of VTE treatment by eliminating the need for an initial parenteral anticoagulant (PAC) as required in conventional therapy..$^{14} \mathrm{~A}$ recent large-scale, real-world evaluation of VTE patients receiving outpatient treatment with apixaban or warfarin in U.S. clinical practice reported that patients treated with apixaban had a significantly lower risk of major bleeding (MB), clinically relevant nonmajor (CRNM) bleeding, and recurrent VTE compared with those treated with warfarin over a mean of 152-day follow-up but without economic assessment. ${ }^{15}$ Furthermore, studies regarding the economic burden of VTE have been conducted but solely within the Medicare fee-for-service population. ${ }^{16}$

The economic burden of warfarin versus apixaban in treatment of VTE has not been well established using commercially insured data in the literature. Therefore, the aim of this study was to compare safety and effectiveness of warfarin versus apixaban and evaluate the associated economic burden in treating VTE patients in a large, commercially insured U.S. population.

\section{Methods}

\section{Data Source}

This retrospective cohort study used the IQVIA PharMetrics Real-World Data Adjudicated Claims (PharMetrics Plus, PMTX+) database from March 1, 2014, to September 30, 2018. PMTX+ data were selected primarily due to national representativeness of the data over other available commercial claims databases. ${ }^{17}$ It provides fully adjudicated health plan claims information for 150 million nonidentified lives in the United States, sourcing information from several commercial health plans in 50 states. It includes patients with $97 \%$ commercial, 2\% Medicare Advantage, and 1\% Medicaid coverage.

\section{Patient Selection}

Patients with a VTE diagnosis claim in any position in inpatient and outpatient (including emergency room) settings were identified from September 1, 2014, to September 30, 2018. The date of the first VTE encounter was designated as the index VTE encounter date. Patients who were aged $\geq 18$ years were selected if they had $\geq 1$ pharmacy claim for warfarin or apixaban on or within the 30-day period following the index VTE encounter. The first warfarin or apixaban prescription date was designated as the index date. Patients were required to have continuous health plan enrollment with medical and pharmacy benefits for 6 months before the index VTE encounter date and during the time between the index VTE encounter and index date. The baseline period was defined as 6 months before the index VTE encounter including the index date.

Patients were excluded if they had atrial fibrillation/atrial flutter or a mechanical heart valve during the baseline period, received oral anticoagulant (OAC) or PAC (unless the therapy was administered prophylactically), or had a VTE diagnosis 6 months before the index VTE encounter. Patients were also excluded if they had evidence of active malignancy (defined as $\geq 2$ medical claims for cancer diagnosis or 1 claim for cancer diagnosis and $\geq 1$ claim for cancer treatment) 6 months before until 30 days after the index VTE encounter, a claim for inferior vena cava filter or pregnancy during the study period or evidence of nonindex OAC use on the index date or a nonindex OAC during the period between the index VTE encounter and the index date. Finally, warfarin patients with their index VTE encounter in the outpatient setting were excluded if they did not have evidence of PAC use (i.e., bridging therapy) within \pm 14 days of the index date or if they received PAC treatment beyond the 14-day period after the index date. Warfarin patients with an inpatient index VTE event were assumed to have bridged therapy with PAC (Figure 1).

The follow-up period was variable and was defined from the day after the index date through the earliest of the following: (a) end of the subsequent 6-month period, (b) health plan disenrollment, (c) index therapy discontinuation, (d) switch to nonindex OAC/PAC treatment, or (e) study end. Discontinuation was defined as no evidence of index warfarin or apixaban use for 30 days from the day of the days supply of the last filled prescription. Switch was defined as a prescription for a nonindex OAC/PAC within 30 days before or after the discontinuation date.

Demographic and clinical characteristics (comorbidities and medication use) and all-cause health care resource utilization and costs were evaluated during the baseline period. Additionally, VTE-related variables, including site of index VTE encounter (inpatient vs. outpatient), VTE diagnosis (DVT 
Comparative Clinical and Economic Outcomes Associated with Warfarin Versus Apixaban in the Treatment of Patients with Venous Thromboembolism in a Large U.S. Commercial Claims Database

\section{FIGURE 1 Patient Selection Criteria}

Claims indicating a VTE diagnosis in primary or secondary position in an inpatient or outpatient setting during the identification period (September 1, 2014-September 30, 2018); the first VTE encounter was designated as the index VTE encounter $\mathrm{N}=504,434$

Claims for apixaban or warfarin during the 30-day period on or after the index VTE encounter during the identification period; the first claim of apixaban or warfarin was designated as the index date $\mathrm{n}=82,601$

Aged $\geq 18$ years and had continuous health plan enrollment with medical and pharmacy benefits for 6 months before or on the index date $\mathrm{n}=58,376$

No claims indicating atrial fibrillation/atrial flutter or mechanical heart valve during the 6 months before or on the index date $\mathrm{n}=51,374$

\section{$\checkmark$}

No pharmacy claim for any OAC during the 6 months before or on the index date or during the period between the index VTE encounter and the index date $n=50,588$

No claims indicating active malignancy 6 months before, or 30 days after, the index VTE encounter; a VTE event during the baseline period; or an inferior vena cava filter or pregnancy during the study period (March 1, 2014-September 30, 2018)

$$
n=29,135
$$

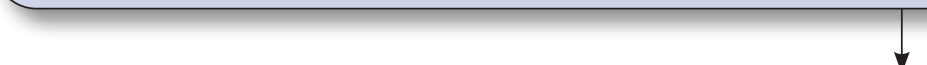

Among warfarin patients with their index VTE encounter in the outpatient setting, patients without evidence of PAC use 14 days before or after the index date were excluded

$n=25,193$

Patients included in the study $(\mathrm{N}=25,193)$

Apixaban $=11,772$

Warfarin $=13,421$

Matched patients

Apixaban/Warfarin $=8,858$

$O A C=$ oral anticoagulant $P A C=$ parenteral anticoagulant VTE $=$ venous thromboembolism.

only vs. PE with or without DVT), position of VTE diagnosis (primary vs. secondary) and VTE etiology (provoked vs. unprovoked) were measured during the index VTE encounter. Provoked VTE was defined as events that are preceded by hormone therapy, fracture/trauma involving lower extremities, pelvic/orthopedic surgery, or hospitalization for any reason for $\geq 3$ days during the 3-month pre-index VTE encounter period $^{18}$; unprovoked VTE was defined as all events not classified as provoked events. ${ }^{18}$

\section{Outcome Measures}

Clinical Outcomes. The clinical outcomes evaluated in this study were MB, CRNM bleeding, recurrent VTE, and all-cause hospitalization. MB was identified using an International Classification of Diseases, Ninth Revision, Clinical Modification (ICD-9-CM) or International Classification of Diseases, 10th Revision, Clinical Modification (ICD-10-CM) diagnosis or procedure code as the primary diagnosis in the inpatient setting and was stratified by gastrointestinal (GI) bleeding; 
intracranial hemorrhage (ICH); and bleeding at other key sites (e.g., hepatic, splenic, and ocular hemorrhage; Appendix A, available in online article).$^{19} \mathrm{CRNM}$ bleeding was identified using secondary diagnosis codes in the inpatient setting for noncritical sites for bleeding (not including patients with MB in the inpatient setting) or a diagnosis code for GI bleeding or other bleeding in the outpatient setting. ${ }^{16}$ Recurrent VTE was defined by primary diagnosis in an inpatient setting, excluding admissions that occurred within 7 days of the index VTE encounter. ${ }^{15}$ All-cause hospitalization was defined as an acute inpatient stay related to any cause at any time during the follow-up period.

Economic Outcomes. Economic outcomes, including all-cause inpatient costs, all-cause emergency room (ER) costs, all-cause office visits costs, all-cause outpatient costs, all-cause medical costs, all-cause pharmacy costs, all-cause health care costs, MB-related costs, and VTE-related costs were evaluated in this study. All-cause inpatient, ER, and office costs were defined as costs related to any inpatient, ER, or office stay respectively during the follow-up. All-cause outpatient costs were defined as the sum of costs in the outpatient, ER, or office setting during the follow-up. All-cause medical costs were defined as the sum of costs in inpatient and outpatient setting during the follow-up. All-cause pharmacy costs included costs for all prescriptions during the follow-up, not just for OAC medications. All-cause health care costs were defined as the sum of medical and pharmacy costs during the follow-up. MB-related medical costs were defined as hospitalization costs associated with the first $\mathrm{MB}$ event plus all subsequent bleeding costs occurring in the inpatient or outpatient setting (primary and secondary diagnoses). Recurrent VTE-related medical costs were defined as the hospitalization costs associated with the first recurrent VTE event plus all subsequent VTE costs in the inpatient or outpatient (primary and secondary diagnoses) setting. Because a fixed follow-up was not used, the total costs were calculated per patient per month (PPPM) and adjusted to 2018 U.S. dollars. The numerator for PPPM calculation was the aggregate of all costs in each category, and the denominator was the aggregated patient-months.

\section{Statistical Methods}

Bivariate comparisons of baseline characteristics were conducted using t-tests and chi-square tests. Propensity score matching (PSM) without replacement was conducted between the warfarin and apixaban cohorts to balance the patient characteristics between cohorts. Patients were matched 1:1 based on the propensity scores generated by logistic regression. Some of the baseline variables included in the PSM were demographics, VTE-related variables, Deyo-Charlson Comorbidity Index (CCI) score, baseline comorbidities, selected surgeries, and baseline medication use (Appendix B, available in online article).$^{20}$ The nearest neighbor method with a caliper of 0.01 was used to match patients.

Covariate balance was checked through standardized differences, with a threshold of $10 \%$; covariates with a standardized difference greater than $10 \%$ were considered unbalanced. ${ }^{21}$ The incidence rates for the clinical outcomes were calculated as the number of events per 100 person-years. The first event per patient for each clinical outcome was included in the calculation. As all variables were balanced after PSM, OAC was the only independent variable included in the models. Cox proportional models were conducted to compare the risk of MB, CRNM bleeding, recurrent VTE, and all-cause hospitalization among the postmatch warfarin and apixaban cohorts.

The all-cause health care costs among the postmatched population were evaluated using generalized linear models with log-link and gamma distribution, wherein cost was the dependent variable.

Two-part models with bootstrapping were implemented for $\mathrm{MB}$ and recurrent VTE-related costs to account for a large number of zeros $(\$ 0)$ because very few patients had MB or recurrent VTE event costs. In the 2-part models, the first part was a logistic regression for the occurrence of the event (MB or recurrent VTE) and the second part was a generalized linear model regression of cost, conditional on the event.. ${ }^{22}$ To mitigate the impact of extreme cost outliers in the data, MB and recurrent VTE-related costs were adjusted using the winsorization method. Winsorization transforms the cost of the outliers to the preestablished percentile of the data. ${ }^{23,24}$ Therefore, the event-related cost values below the 5 th percentile were winsorized to costs in the 5 th percentile, and costs above the 95th percentile were transformed to costs in the 95th percentile.

\section{Sensitivity Analysis}

There is variation in the literature surrounding the definition of recurrent VTE. ${ }^{916,25-28}$ Therefore, to test the robustness of our findings, 3 sensitivity analyses were conducted surrounding the definition of recurrent VTE for both clinical and economic outcomes. First, recurrent VTE was defined to include events occurring within 7 days of the qualifying index VTE encounter. ${ }^{26}$ Second, recurrent VTE was defined to include encounters in the inpatient and emergency room (ER) settings, excluding events within 7 days of the index VTE encounter. ${ }^{28}$ Third, recurrent VTE was defined to include both encounters within 7 days of the index VTE encounter and those occurring in the ER setting. ${ }^{16}$

\section{Results}

\section{Baseline Characteristics}

Among 25,193 prematched patients, 13,421 (53.3\%) were prescribed warfarin, and 11,772 (46.7\%) were prescribed apixaban 
Comparative Clinical and Economic Outcomes Associated with Warfarin Versus Apixaban in the Treatment of Patients with Venous Thromboembolism in a Large U.S. Commercial Claims Database

\section{TABLE 1 Prematched and Postmatched Descriptive Baseline Characteristics Among VTE Patients Prescribed} Warfarin Versus Apixaban

\begin{tabular}{|c|c|c|c|c|c|c|}
\hline & & Prematched & & & ostmatched & \\
\hline & $\begin{array}{l}\text { Apixaban Cohort } \\
\text { (Reference) }\end{array}$ & Warfarin Cohort & STD $^{\mathrm{a}}$ & $\begin{array}{c}\text { Apixaban Cohort } \\
\text { (Reference) }\end{array}$ & Warfarin Cohort & STD $^{\mathrm{a}}$ \\
\hline Sample size & 11,772 & 13,421 & & 8,858 & 8,858 & \\
\hline Age, years, mean (SD) & $52.7(12.7)$ & $52.4(13.1)$ & 1.90 & $52.6(12.9)$ & $52.5(12.9)$ & 0.84 \\
\hline Age, years, n (\%) & & & & & & \\
\hline $18-54$ & $5,956 \quad(50.6)$ & $6,946 \quad(51.8)$ & 2.33 & $4,527 \quad(51.1)$ & $4,538 \quad(51.2)$ & 0.25 \\
\hline $55-64$ & $4,252 \quad(36.1)$ & $4,698 \quad(35.0)$ & 2.34 & $3,162 \quad(35.7)$ & $3,157 \quad(35.6)$ & 0.12 \\
\hline $65-74$ & $1,144 \quad(9.7)$ & $1,214 \quad(9.0)$ & 2.30 & $815 \quad(9.2)$ & $(9.4)$ & 0.58 \\
\hline $75-79$ & $155 \quad(1.3)$ & $(1.5)$ & 1.84 & $(1.5)$ & $(1.4)$ & 0.96 \\
\hline$\geq 80$ & $(2.3)$ & $(2.7)$ & 2.64 & $(2.5)$ & $(2.4)$ & 0.80 \\
\hline Gender, n (\%) & & & & & & \\
\hline Male & $6,554 \quad(55.7)$ & $7,214 \quad(53.8)$ & 3.85 & $4,907 \quad(55.4)$ & $4,910 \quad(55.4)$ & 0.07 \\
\hline Female & $5,218 \quad(44.3)$ & $6,207 \quad(46.2)$ & 3.87 & $3,951 \quad(44.6)$ & $3,948 \quad(44.6)$ & 0.07 \\
\hline Geographic region, n (\%) & & & & & & \\
\hline Northeast & $2,281 \quad(19.4)$ & $2,713 \quad(20.2)$ & 2.11 & $1,993 \quad(22.5)$ & $1,918 \quad(21.7)$ & 2.04 \\
\hline North Central & $2,756 \quad(23.4)$ & $4,348 \quad(32.4)$ & 20.12 & $2,631 \quad(29.7)$ & $2,643 \quad(29.8)$ & 0.30 \\
\hline South & $5,216 \quad(44.3)$ & $3,194 \quad(23.8)$ & 44.32 & $2,732 \quad(30.8)$ & $2,815 \quad(31.8)$ & 2.02 \\
\hline West & $1,427 \quad(12.1)$ & $2,990 \quad(22.3)$ & 27.16 & $1,413 \quad(16.0)$ & $1,401 \quad(15.8)$ & 0.37 \\
\hline Other & $92 \quad(0.8)$ & $176 \quad(1.3)$ & 5.21 & $89 \quad(1.0)$ & $81 \quad(0.9)$ & 0.93 \\
\hline Type of index VTE encounter, $\mathrm{n}(\%)$ & & & & & & \\
\hline Inpatient & $4,568 \quad(38.8)$ & $7,494 \quad(55.8)$ & 34.61 & $4,017 \quad(45.3)$ & $4,113 \quad(46.4)$ & 2.17 \\
\hline Outpatient & $7,204 \quad(61.2)$ & $5,927 \quad(44.2)$ & 34.61 & $4,841 \quad(54.7)$ & $4,745 \quad(53.6)$ & 2.17 \\
\hline Index VTE diagnosis, n (\%) & & & & & & \\
\hline DVT only & $6,455 \quad(54.8)$ & $7,443 \quad(55.5)$ & 1.26 & $4,947 \quad(55.8)$ & $4,879 \quad(55.1)$ & 1.54 \\
\hline PE with or without DVT & $5,317 \quad(45.2)$ & $5,978 \quad(44.5)$ & 1.26 & $3,911 \quad(44.2)$ & $3,979 \quad(44.9)$ & 1.54 \\
\hline Index VTE etiology, n (\%) & & & & & & \\
\hline Provoked & $3,545 \quad(30.1)$ & $4,335 \quad(32.3)$ & 4.73 & $2,709 \quad(30.6)$ & $2,741 \quad(30.9)$ & 0.78 \\
\hline Unprovoked & $8,227 \quad(69.9)$ & $9,086 \quad(67.7)$ & 4.73 & $6,149 \quad(69.4)$ & $6,117 \quad(69.1)$ & 0.78 \\
\hline Deyo-Charlson Comorbidity Index, mean (SD) & $0.8 \quad(1.4)$ & $1.0 \quad(1.6)$ & 13.60 & $0.8 \quad(1.5)$ & $0.8 \quad(1.5)$ & 0.90 \\
\hline Baseline comorbidity, n (\%) & & & & & & \\
\hline Anemia & $1,720 \quad(14.6)$ & $2,715 \quad(20.2)$ & 14.83 & $1,417 \quad(16.0)$ & $1,422(16.1)$ & 0.15 \\
\hline Central venous catheter & $721 \quad(6.1)$ & $1,253 \quad(9.3)$ & 12.05 & $628 \quad(7.1)$ & $633 \quad(7.1)$ & 0.22 \\
\hline Cerebrovascular disease & $547 \quad(4.6)$ & $979 \quad(7.3)$ & 11.19 & $461 \quad(5.2)$ & $456 \quad(5.1)$ & 0.25 \\
\hline Ischemic heart/coronary artery disease & $1,467 \quad(12.5)$ & $1,746 \quad(13.0)$ & 1.65 & $1,068 \quad(12.1)$ & $1,076 \quad(12.1)$ & 0.28 \\
\hline Dyspepsia or stomach discomfort & $2,236 \quad(19.0)$ & $2,929 \quad(21.8)$ & 7.03 & $1,732 \quad(19.6)$ & $1,732 \quad(19.6)$ & 0.00 \\
\hline Hyperlipidemia & $3,498 \quad(29.7)$ & $3,819 \quad(28.5)$ & 2.77 & $2,519(28.4)$ & $2,523(28.5)$ & 0.10 \\
\hline Obesity & $3,246 \quad(27.6)$ & $3,814 \quad(28.4)$ & 1.87 & $2,403 \quad(27.1)$ & $2,451 \quad(27.7)$ & 1.21 \\
\hline Pneumonia & $1,211 \quad(10.3)$ & $1,612 \quad(12.0)$ & 5.45 & $914 \quad(10.3)$ & $939 \quad(10.6)$ & 0.92 \\
\hline Sleep apnea & $1,432(12.2)$ & 1,728 (12.9) & 2.15 & $1,084 \quad(12.2)$ & $1,089 \quad(12.3)$ & 0.17 \\
\hline Thrombophilia & $537 \quad(4.6)$ & $908 \quad(6.8)$ & 9.55 & $468 \quad(5.3)$ & $475 \quad(5.4)$ & 0.35 \\
\hline Congestive heart failure & $794 \quad(6.7)$ & $1,200 \quad(8.9)$ & 8.14 & $634 \quad(7.2)$ & (7.4) & 0.96 \\
\hline Diabetes & $2,023 \quad(17.2)$ & $2,679 \quad(20.0)$ & 7.15 & $1,569 \quad(17.7)$ & 1,563 & 0.18 \\
\hline Hypertension & $5,509 \quad(46.8)$ & $6,479 \quad(48.3)$ & 2.97 & $4,055 \quad(45.8)$ & $4,062 \quad(45.9)$ & 0.16 \\
\hline Renal disease & $977 \quad(8.3)$ & $1,500 \quad(11.2)$ & 9.72 & $795 \quad(9.0)$ & $(9.3)$ & 1.14 \\
\hline Liver disease & $(7.3)$ & $1,169 \quad(8.7)$ & 5.05 & $(7.4)$ & $(7.5)$ & 0.52 \\
\hline COPD & $(7.7)$ & 1,199 & 4.29 & $(8.1)$ & $(7.9)$ & 0.71 \\
\hline Peripheral vascular disease & $(7.4)$ & 1,302 & 8.27 & $(7.8)$ & $(7.9)$ & 0.42 \\
\hline Baseline bleed & $1,612 \quad(13.7)$ & $2,677 \quad(19.9)$ & 16.78 & $1,362(15.4)$ & $1,366 \quad(15.4)$ & 0.13 \\
\hline Fracture/trauma involving lower extremities, n (\%) & $1,532(13.0)$ & $1,783 \quad(13.3)$ & 0.81 & $1,157 \quad(13.1)$ & $1,182 \quad(13.3)$ & 0.83 \\
\hline Selected surgeries, $\mathrm{n}(\%)$ & $2,528 \quad(21.5)$ & $3,540(26.4)$ & 11.51 & $1,992(22.5)$ & $2,037 \quad(23.0)$ & 1.21 \\
\hline
\end{tabular}

aSTD: standardized difference $=100 \times$ (actual STD). A standardized difference greater than 10 is considered significant.

$C O P D=$ chronic obstructive pulmonary disease; $D V T=$ deep vein thrombosis; $P E=$ pulmonary embolism; $S D=$ standard deviation; VTE =venous thromboembolism. 
Comparative Clinical and Economic Outcomes Associated with Warfarin Versus Apixaban in the Treatment of Patients with Venous Thromboembolism in a Large U.S. Commercial Claims Database

FIGURE 2 Propensity Score-Matched Incidence Rates and Hazard Ratios of Major Bleeding, CRNM Bleeding, Recurrent VTE, and All-Cause Hospitalization for Warfarin Versus Apixaban

\begin{tabular}{|c|c|c|c|c|c|}
\hline & $\begin{array}{c}\text { Warfarin } \\
\text { Events, } \mathrm{n} \text { (inciden }\end{array}$ & $\begin{array}{l}\text { Apixaban } \\
\text { r } 100 \text { person }\end{array}$ & & $\begin{array}{l}\text { Hazard Ratio } \\
(95 \% \mathrm{Cl})\end{array}$ & $P$ Value \\
\hline Major bleeding & $117 \quad(4.4)$ & $75 \quad(3.0)$ & $\longrightarrow$ & $1.52(1.14-2.04)$ & 0.005 \\
\hline Gl bleeding & $42(1.6)$ & $24(1.0)$ & $\longrightarrow$ & $1.71(1.04-2.83)$ & 0.036 \\
\hline $\mathrm{ICH}$ & $6 \quad(0.2)$ & $7 \quad(0.3)$ & & $0.84(0.28-2.50)$ & 0.754 \\
\hline Other bleeding & $74 \quad(2.8)$ & $47 \quad(1.9)$ & $\longrightarrow$ & $1.54(1.06-2.22)$ & 0.022 \\
\hline CRNM bleeding & $862(34.6)$ & $674(28.2)$ & $\rightarrow$ & $1.27(1.15-1.40)$ & $<0.001$ \\
\hline GI bleeding & $195 \quad(7.4)$ & $186 \quad(7.5)$ & $=$ & $1.02(0.84-1.25)$ & 0.836 \\
\hline Other bleeding & $678(26.8)$ & $494(20.4)$ & $\rightarrow$ & $1.36(1.21-1.53)$ & $<0.001$ \\
\hline Recurrent VTE & $263 \quad(9.9)$ & $175 \quad(7.0)$ & $\rightarrow-$ & $1.50(1.24-1.82)$ & $<0.001$ \\
\hline All-cause hospitalization & $948(38.0)$ & $861(36.0)$ & $\rightarrow$ & $1.08(0.98-1.18)$ & 0.113 \\
\hline
\end{tabular}

Note: In this figure, apixaban was used as the reference.

$C I=$ confidence interval; $C R N M=$ clinically relevant nonmajor; $G I=$ gastrointestinal; $I C H=$ intracranial hemorrhage; VTE=venous thromboembolism.

(Figure 1). Patients prescribed warfarin had higher mean CCI scores, more selected surgeries, and more history of falls during the baseline period compared with apixaban in the prematched population (Appendix B, available in online article). After 1:1 PSM, 8,858 warfarin-apixaban pairs were selected with a mean follow-up of 109 days and 103 days, respectively (Table 1).

After PSM, the standardized differences of the means of the matched variables were less than $10 \%$, indicating that the covariates were balanced between treatment groups at baseline. Apixaban- and warfarin-matched patients had a mean age of 52.5 years and a mean CCI score of 0.8 . Approximately $46 \%$ of patients had an index VTE encounter in the inpatient setting, 55\% of patients had a diagnosis of DVT only, and 31\% of patients had a provoked VTE diagnosis (Table 1).

\section{Clinical Outcomes}

In the PSM-matched population, the incidence rates of MB (4.4 vs. 3.0 per 100 person-years), CRNM bleeding (34.6 vs. 28.2 per 100 person-years), and recurrent VTE (9.9 vs. 7.0 per 100 person-years; Figure 2) were higher for warfarin compared with apixaban. Warfarin and apixaban patients had a similar incidence of all-cause hospitalization during the follow-up period (38.0 vs. 36.0 per 100 person-years).

Warfarin was associated with a significantly higher risk of $\mathrm{MB}$ (hazard ratio $[\mathrm{HR}]=1.52,95 \%$ confidence interval
$[C I]=1.14-2.04 ; P=0.005), C R N M$ bleeding $(H R=1.27,95 \%$ $\mathrm{CI}=1.15-1.40 ; P<0.001)$, and recurrent VTE $(\mathrm{HR}=1.50,95 \%$ $\mathrm{CI}=1.24-1.82 ; \mathrm{P}<0.001)$ compared with apixaban. The risk of all-cause hospitalization was similar for warfarin patients compared with apixaban patients $(\mathrm{HR}=1.08,95 \% \mathrm{CI}=0.98-1.18$; $P=0.113$; Figure 2).

\section{Economic Outcomes}

Warfarin patients had significantly higher all-cause medical costs PPPM ( $\$ 2,333$ vs. $\$ 1,992 ; P=0.001)$ compared with apixaban patients. After pharmacy costs were included, warfarin patients had similar all-cause total health care costs PPPM ( $\$ 2,630$ vs. $\$ 2,420 ; P=0.051$; Table 2) compared with apixaban patients. Warfarin was however associated with significantly higher MB-related medical costs PPPM ( $\$ 112$ vs. $\$ 65$; difference $=\$ 47,95 \% \mathrm{CI}=\$ 8-\$ 90 ; P=0.020$; Table 2) and recurrent VTE-related medical costs PPPM (\$287 vs. \$206; difference $=\$ 82,95 \% \mathrm{CI}=\$ 17-\$ 145 ; P=0.014)$ compared with apixaban patients.

\section{Sensitivity Analyses}

Both the clinical and economic outcomes from all 3 sensitivity analyses were consistent with the main analysis (Table 3). Recurrent VTE incidence and recurrent VTE-related costs were significantly higher in warfarin patients compared with apixaban patients when recurrent VTE was defined to include events 
Comparative Clinical and Economic Outcomes Associated with Warfarin Versus Apixaban in the Treatment of Patients with Venous Thromboembolism in a Large U.S. Commercial Claims Database

TABLE 2 All-Cause Health Care Costs in the Propensity Score-Matched Cohorts

\begin{tabular}{|c|c|c|c|c|c|c|c|c|c|c|}
\hline & \multicolumn{3}{|c|}{ Apixaban (Reference) } & \multicolumn{3}{|c|}{ Warfarin } & \multirow{2}{*}{$\begin{array}{c}\text { Difference } \\
\text { Between } \\
\text { Marginal } \\
\text { Effects }\end{array}$} & \multirow{2}{*}{\multicolumn{2}{|c|}{$\begin{array}{c}95 \% \text { CI for } \\
\text { Difference Between } \\
\text { Marginal Effect }\end{array}$}} & \multirow[b]{2}{*}{$P$ Value } \\
\hline & $\begin{array}{l}\text { Marginal } \\
\text { Effect }\end{array}$ & \multicolumn{2}{|c|}{$\begin{array}{l}\text { 95\% CI for } \\
\text { Marginal Effect }\end{array}$} & $\begin{array}{l}\text { Marginal } \\
\text { Effect }\end{array}$ & \multicolumn{2}{|c|}{$\begin{array}{c}95 \% \text { CI for } \\
\text { Marginal Effect } \\
\end{array}$} & & & & \\
\hline \multicolumn{11}{|c|}{ All-cause health care costs (PPPM), USD } \\
\hline ER visit costs & 73 & 71 & 74 & 102 & 97 & 108 & 30 & 15 & 43 & $<0.001$ \\
\hline Office visit costs & 160 & 157 & 163 & 187 & 178 & 196 & 27 & 21 & 32 & $<0.001$ \\
\hline Other outpatient costs & 829 & 812 & 846 & 974 & 926 & 1,024 & 145 & 56 & 233 & 0.001 \\
\hline $\begin{array}{l}\text { Total outpatient costs } \\
\text { (ER + office + other })\end{array}$ & 1,062 & 1,040 & 1,084 & 1,263 & 1,201 & 1,328 & 201 & 106 & 294 & $<0.001$ \\
\hline Inpatient stay costs & 930 & 910 & 949 & 1,070 & 1,017 & 1,125 & 140 & -33 & 314 & 0.112 \\
\hline $\begin{array}{l}\text { Total medical costs } \\
\text { (outpatient + inpatient) }\end{array}$ & 1,992 & 1,951 & 2,034 & 2,333 & 2,218 & 2,453 & 341 & 134 & 548 & 0.001 \\
\hline Pharmacy costs & 429 & 420 & 438 & 297 & 283 & 312 & -132 & -162 & -102 & $<0.001$ \\
\hline $\begin{array}{l}\text { Total health care costs } \\
\text { (outpatient }+ \text { inpatient }+ \text { pharmacy) }\end{array}$ & 2,420 & 2,371 & 2,471 & 2,630 & 2,501 & 2,766 & 210 & -1 & 419 & 0.051 \\
\hline $\begin{array}{l}\text { Major bleeding-related medical } \\
\text { costs (PPPM), USD }\end{array}$ & 65 & 63 & 67 & 112 & 109 & 116 & 47 & 8 & 90 & 0.020 \\
\hline $\begin{array}{l}\text { Recurrent VTE-related medical } \\
\text { costs (PPPM), USD }\end{array}$ & 206 & 203 & 209 & 287 & 283 & 292 & 82 & 17 & 145 & 0.014 \\
\hline
\end{tabular}

within 7 days of the index VTE encounter, events in the ER setting, and events both within 7 days of index VTE encounter and the ER setting in aggregate.

\section{Discussion}

This retrospective cohort analysis evaluated the clinical and economic burden associated with VTE among patients treated with warfarin and apixaban in a younger, commercially insured population compared with the published literature. ${ }^{15,16}$ Warfarin was associated with a higher risk of MB, CRNM bleeding, and recurrent VTE among VTE patients compared with patients prescribed apixaban. In addition to observed clinical benefit, warfarin use was also associated with higher all-cause medical costs PPPM, and MB- and recurrent VTE-related costs PPPM compared with apixaban use. All-cause health care costs were similar among warfarin and apixaban patients.

The results from this study add evidence to supplement results from the AMPLIFY trial where a benefit of reduced risk of $\mathrm{MB}(0.6 \%$ vs. $1.8 \%$; relative risk $=0.31[0.17-0.55])$ and CRNM bleeding (3.8\% vs. 8.0\%; relative risk $=0.48$ [0.38-0.60]) was observed when patients were treated with apixaban compared with conventional therapy (subcutaneous enoxaparin followed by warfarin). ${ }^{13}$ The trial also reported a reduced risk of recurrent VTE in apixaban patients; however, the association was not statistically significant $(\mathrm{HR}=0.84,95 \% \mathrm{CI}=0.60-1.18)$. In this study, in addition to MB and CRNM bleeding, recurrent VTE was also significantly higher for warfarin- compared with apixaban-treated patients. The inconsistency in the recurrent VTE risk could be attributed to study setting (controlled trial vs. real world), differences in the methods employed to identify eligible VTE patients (diagnoses via imaging techniques vs. ICD-9-CM and ICD-10-CM codes), and variable follow-up periods. and definition of recurrent VTE (clinical assessment including fatal and nonfatal VTE vs. a primary diagnosis in an inpatient setting). Additionally, the AMPLIFY trial was designed to assess noninferiority in a clinical trial setting, whereas the present real-world study has a much larger sample size for detecting association.

Other retrospective studies comparing DOACs to warfarin have shown similar results. ${ }^{15,19}$ Using a commercial pooled database, VTE patients who initiated apixaban were associated with a significantly lower risk of $\mathrm{MB}(\mathrm{HR}=0.75,95 \% \mathrm{CI}=0.64$ 0.87), CRNM bleeding ( $\mathrm{HR}=0.77,95 \% \mathrm{CI}=0.71-0.83$ ), and recurrent VTE $(\mathrm{HR}=0.80,95 \% \mathrm{CI}=0.70-0.91)$ compared with warfarin patients, which is consistent with our study. ${ }^{15}$ In another study using a Medicare population, warfarin was associated with a significantly higher risk of $\mathrm{MB}(\mathrm{HR}=1.31$, 95\% CI $=1.10-1.57)$ and CRNM bleeding ( $\mathrm{HR}=1.31,95 \%$ $\mathrm{CI}=1.19-1.43 ; \mathrm{P}<0.001)$, but a similar risk of recurrent VTE $(\mathrm{HR}=0.96,95 \% \mathrm{CI}=0.70-1.33 ; P=0.825)$, compared with apixaban patients. ${ }^{19}$ Although the $\mathrm{MB}$ and CRNM bleeding risks are consistent with the Medicare study, the risk of recurrent VTE is not. This could be attributed to the different health insurances (i.e., Medicare vs. commercial insurance) or the differences in 
Comparative Clinical and Economic Outcomes Associated with Warfarin Versus Apixaban in the Treatment of Patients with Venous Thromboembolism in a Large U.S. Commercial Claims Database

TABLE 3 Sensitivity Analysis for Clinical and Economic Outcomes for Recurrent VTE

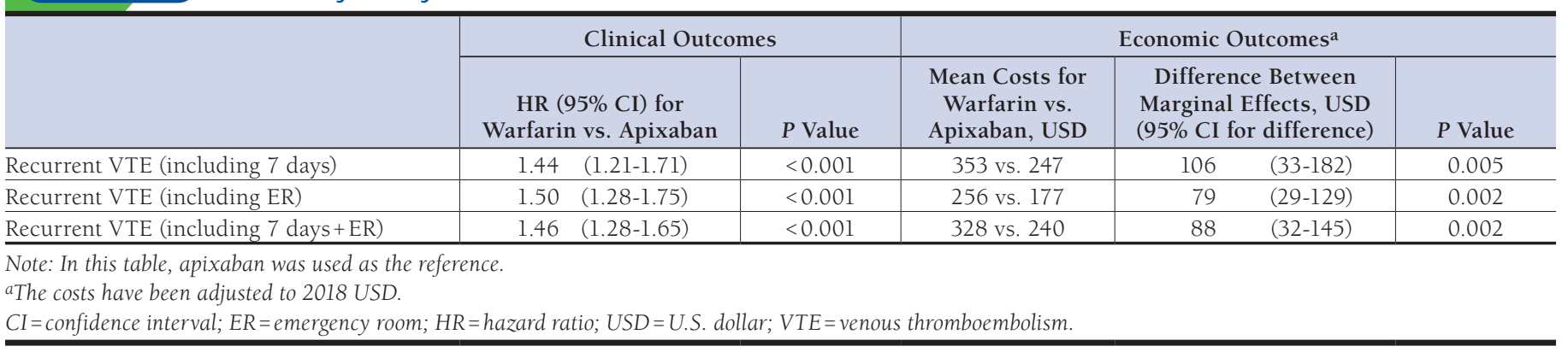

the data structure. The Medicare study also included higherrisk older patients with a mean age of 78 years, compared with a younger population with a mean age of 52 years in this study. Additionally, the current study used more recent data (until September 2018) compared with the Medicare study (until December 2016), which could have affected the outcomes.

Fewer studies have evaluated the economic burden as well as the impact of complications including recurrence of VTE and $\mathrm{MB}$ among patients who initiated oral anticoagulants in a younger, commercially insured VTE population. Lin et al. (2014) found that among Medicare and commercially insured VTE patients, recurrent VTE rates were relatively high (11.4\% and $15.4 \%$, respectively). ${ }^{9}$ A systematic review had estimated that managing an initial episode of acute VTE costs between $\$ 12,000$ and $\$ 15,000$, with subsequent complications increasing costs to between $\$ 18,000$ and 23,000 per incident case. ${ }^{10}$ Additionally, the authors found that the primary driver for health care costs was the use of inpatient care, which is similar to the findings of the present study. ${ }^{10}$ They concluded that among the VTE patient population, the total health care costs for patients with a recurrent VTE event were approximately triple those for patients who did not experience a recurrent VTE event. ${ }^{9}$ The above-mentioned study using the Medicare population found that all-cause $(\$ 3,267$ vs. $\$ 3,033 ; P<0.001)$ and MB-related costs ( $\$ 147$ vs. $\$ 75 ; P=0.003$ ) were significantly higher for warfarin patients whereas recurrent VTE related costs were similar ( $\$ 30$ vs. $\$ 36 ; P=0.516$ ) compared with apixaban patients. ${ }^{19}$ In this study, warfarin patients had significantly higher MB- and recurrent VTE-related costs compared with apixaban patients using winsorization. A similar trend was seen without applying winsorization; warfarin trended toward higher MB- and recurrent VTE-related costs; however, the MB-related costs were not significant. One likely reason for this may be due to the extreme cost outliers and the smaller sample size associated with MB and recurrent VTE events.

\section{Limitations}

To the best of our knowledge, this retrospective analysis is the first study that evaluated economic burden associated with VTE and recurrence of VTE in a commercially insured population. It provides a comprehensive picture of how medications are used by clinicians in routine practice. One of the main strengths of the study is that it has a wide geographic U.S. representation and can be generalized to the U.S. commercially insured population younger than aged 65 years.

There are some inherent limitations to this study and the nature of observational research. Causal relationships cannot be reported; only associations could be assessed. Additionally, administrative claims data are collected for purposes other than research, and the analysis is constrained by diagnosis and procedure codes. The presence of a diagnosis code on a medical claim is not a positive indication of the presence of disease, as the diagnosis code may be incorrectly coded or included as rule-out criteria rather than actual disease. Over-the-counter use of medications such as aspirin could not be captured which could affect the treatment patterns associated with the study anticoagulants.

This study used ICD-9-CM and ICD-10-CM codes to identify DVT and PE, which is different from the imaging assessments used in clinical trials; this may lead to differences in patient populations. Also, although cohorts were matched using PSM, there remains the potential for residual confounding, such as warfarin dose adjustment and over-the-counter use of aspirin, which was not available in the database. Finally, these results may not be generalizable to those who are uninsured, the Medicare population, or other segments of the U.S. population.

\section{Conclusions}

In this commercially insured population, warfarin use among VTE patients was associated with higher risk of MB, CRNM bleeding, and recurrent VTE compared with apixaban use. 


\section{Comparative Clinical and Economic Outcomes Associated with Warfarin Versus Apixaban in the Treatment of Patients with Venous Thromboembolism in a Large U.S. Commercial Claims Database}

These results are largely consistent with available clinical trial and recently published real-world data. Warfarin was also associated with higher medical health care costs, higher MB-related costs, and higher recurrent VTE-related costs compared with apixaban. These results may help clinicians, stakeholders, and decision makers in identifying safe and effective treatment for VTE as well as potentially reducing economic burden.

\section{Authors}

JENNIFER D. GUO, PhD; JIGAR RAJPURA, PhD; GARY J OKANO, PhD; and LISA ROSENBLATT, MD, Bristol Myers Squibb, Lawrenceville, New Jersey. PATRICK HLAVACEK, MPH; JACK MARDEKIAN, PhD; and CRISTINA RUSS, MD, Pfizer, New York, New York. ALLISON KESHISHIAN, MPH; JANVI SAH, MS; RACHEL DELINGER, MPH; and QIAO MU, MPH, SIMR, LLC, Ann Arbor, Michigan.

AUTHOR CORRESPONDENCE: Jigar Rajpura, PhD, Bristol Myers Squibb, 3551 Lawrenceville Rd., Lawrenceville, NJ 08648.

Tel.: 551.689.4595; Email: jigar.rajpura@bms.com.

\section{DISCLOSURES}

This study was funded by Bristol Myers Squibb and Pfizer, which were also involved in the study design, as well as writing and revising of the manuscript. Guo, Rajpura, Okano, and Rosenblatt are employees of Bristol Myers Squibb. Hlavacek, Mardekian, and Russ are employees of Pfizer. Keshishian, Sah, Delinger, and Mu are employees of SIMR, LLC, which received funding from the study sponsors to conduct this study.

\section{ACKNOWLEDGMENTS}

The editorial support provided by Christopher Haddlesey at SIMR, LLC, was funded by Bristol Myers Squibb and Pfizer.

\section{REFERENCES}

1. Nielsen JD. The incidence of pulmonary embolism during deep vein thrombosis. Phlebology. 2013;28(Suppl 1):29-33.

2. Goldhaber SZ, Bounameaux H. Pulmonary embolism and deep vein thrombosis. Lancet. 2012;379(9828):1835-46.

3. Lehmann R, Luxembourg B, Miesbach W, et al. Idiopathic (unexplained) pulmonary embolism is associated with an impaired prognosis compared to other entities of pulmonary embolism. Blood Coagul Fibrinolysis. 2010;21(1):70-76

4. Kim DY, Kobayashi L, Barmparas G, Fortlage D, Curry T, Coimbra R. Venous thromboembolism in the elderly: the result of comorbid conditions or a consequence of injury? J Trauma Acute Care Surg. 2012;72(5):1286-91.

5. Velmahos GC, Spaniolas K, Tabbara M, et al. Pulmonary embolism and deep venous thrombosis in trauma: are they related? Arch Surg. 2009;144(10):928-32.

6. Wilasrusmee C, Kiranantawat K, Horsirimanont S, et al. Deep venous thrombosis in surgical intensive care unit: prevalence and risk factors. Asian J Surg. 2009;32(2):85-88.
7. Darze ES, Latado AL, Guimarães AG, et al. Incidence and clinical predictors of pulmonary embolism in severe heart failure patients admitted to a coronary care unit. Chest. 2005;128(4):2576-80.

8. Cushman M, Kuller LH, Prentice R, et al. Estrogen plus progestin and risk of venous thrombosis. JAMA. 2004;292(13):1573-80.

9 Lin J, Lingohr-Smith M, Kwong WJ. Incremental health care resource utilization and economic burden of venous thromboembolism recurrence from a U.S. payer perspective. J Manag Care Pharm. 2014;20(2):174-86. Available at: https://www.jmcp.org/doi/10.18553/jmcp.2014.20.2.174.

10. Grosse SD, Nelson RE, Nyarko KA, Richardson LC, Raskob GE. The economic burden of incident venous thromboembolism in the United States: a review of estimated attributable healthcare costs. Thromb Res. 2016;137:3-10.

11. Mahan CE, Borrego ME, Woersching AL, et al. Venous thromboembolism: annualised United States models for total, hospital-acquired and preventable costs utilising long-term attack rates. Thromb Haemost. 2012;108(2):291-302.

12. Kearon C, Akl EA, Ornelas J, et al. Antithrombotic therapy for VTE disease: CHEST Guideline and Expert Panel Report. Chest. 2016;149(2):315-52.

13. Agnelli G, Buller HR, Cohen A, et al. Oral apixaban for the treatment of acute venous thromboembolism. N Engl J Med. 2013;369(9):799-808.

14. Bauersachs R, Berkowitz SD, Brenner B, et al. Oral rivaroxaban for symptomatic venous thromboembolism. N Engl J Med. 2010;363(26):2499-510.

15. Weycker D, Li X, Wygant GD, et al. Effectiveness and safety of apixaban versus warfarin as outpatient treatment of venous thromboembolism in U.S. clinical practice. Thromb Haemost. 2018;118(11):1951-61.

16. Hlavacek P, Guo J. D, Rosenblatt L, et al. Safety, effectiveness, and health care cost comparisons among elderly patients with venous thromboembolism prescribed warfarin or apixaban in the United States Medicare population. Curr Med Res Opin. 2019;35(12):2043-51

17. Cepeda MS, Fife D, Denarié M, Bradford D, Roy S, Yuan Y Quantification of missing prescriptions in commercial claims databases: results of a cohort study. Pharmacoepidemiol Drug Saf. 2017;26(4):386-92.

18. Kearon C, Ageno W, Cannegieter SC, Cosmi B, Geersing GJ, Kyrle PA; Subcommittees on Control of Anticoagulation, and Predictive and Diagnostic Variables in Thrombotic Disease. Categorization of patients as having provoked or unprovoked venous thromboembolism: guidance from the SSC of ISTH. J Thromb Haemost. 2016;14(7):1480-83.

19. Lip GY, Keshishian A, Li X, et al. Effectiveness and safety of oral anticoagulants among nonvalvular atrial fibrillation patients: the ARISTOPHANES study. Stroke. 2018;49(12):2933-44.

20. Austin PC. The use of propensity score methods with survival or timeto-event outcomes: reporting measures of effect similar to those used in randomized experiments. Stat Med. 2014;33(7):1242-58.

21. Austin PC. Balance diagnostics for comparing the distribution of baseline covariates between treatment groups in propensity-score matched samples. Stat Med. 2009;28(25):3083-107.

22. Lachenbruch PA. Comparisons of two-part models with competitors. Stat Med. 2001;20(8):1215-34

23. Thomas JW, Ward K. Economic profiling of physician specialists: use of outlier treatment and episode attribution rules. Inquiry. 2006;43(3):271-82.

24. Weichle T, Hynes DM, Durazo-Arvizu R, Tarlov E, Zhang Q. Impact of alternative approaches to assess outlying and influential observations on health care costs. Springerplus. 2013;2(1):614.

25. Chen SY, Wu N, Gulseth M, et al. One-year adherence to warfarin treatment for venous thromboembolism in high-risk patients and its association with long-term risk of recurrent events. J Manag Care Pharm. 2013;19(4):291301. Available at: https://www.jmcp.org/doi/10.18553/jmcp.2013.19.4.291.

26. Deitelzweig SB, Lin J, Kreilick C, et al. Warfarin therapy in patients with venous thromboembolism: patterns of use and predictors of clinical outcomes. Adv Ther. 2010;27(9):623-33. 
Comparative Clinical and Economic Outcomes Associated with Warfarin Versus Apixaban in the Treatment of Patients with Venous Thromboembolism in a Large U.S. Commercial Claims Database

27. Willey VJ, Bullano MF, Hauch O, et al. Management patterns and outcomes of patients with venous thromboembolism in the usual community practice setting. Clin Ther. 2004;26(7):1149-59.

28. Cai J, Preblick R, Zhang Q, Kwong WJ. Utilization of parenteral anticoagulants and warfarin: impact on the risk of venous thromboembolism recurrence in the outpatient setting. Am Health Drug Benefits. 2014;7(8):444-51. 


\section{APPENDIX A Codes for Identifying Clinical Outcomes}

\section{Category \\ Major bleeding}

Gastrointestinal

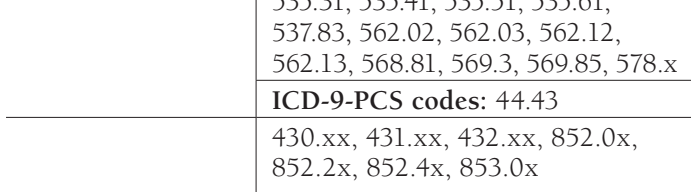

Intracranial

ICD-9-Diagnosis: 285.1, 360.43, 362.43, 362.81, 363.61, 363.62, 363.72, 364.41, 372.72, 374.81, $376.32,377.42,379.23,423.0 x$ $596.7 x, 599.7 x, 602.1 x, 620.1$, $621.4,626.2,626.5,626.7,626.8$, 626.9, 719.1x, 782.7, 784.7, 784.8,

Other major bleeding

\section{ICD-10-PCS codes: no codes}

I6000, I6001, I6002, I6010, I6011, I6012, I602, I6030, I6031, I6032, I604, I6050, I6051, I6052, I606, I607, I608, I609, I610, I611, I612, I613, I614, I615, I616, I618, I619, I6200, I6201, I6202, I6203, I621, I629, S06340A, S06341A, S06342A, S06343A, S06344A, S06345A, S06346A, S06347A, S06348A, S06349A, S06350A, S06351A, S06352A, S06353A, S06354A, S06355A, S06356A, S06357A, S06358A, S06359A, S06360A, S06361A, S06362A, S06363A, S06364A, S06365A, S06366A, S06367A, S06368A, S06369A, S064X0A, S064X1A, S064X2A, S064X3A, S064X4A, S064X5A, S064X6A, S064X7A, S064X8A, S064X9A, S065X0A, S065X1A, S065X2A, S065X3A, S065X4A, S065X5A, S065X6A, S065X7A, S065X8A, S065X9A, S066X0A, S066X1A, S066X2A, S066X3A, S066X4A, S066X5A, S066X6A, S066X7A, S066X8A, S066X9A

ICD-10-CM codes: D62, D7801, D7802, D7821, D7822, E3601, E3602, E89810, E89811, G9731, G9732, G9751, G9752, H05231, H05232, H05233, H05239, H1130, H1131, H1132, H1133, H2100, H2101, H2102, H2103, H31301, H31302, H31303, H31309, H31311, H31312, H31313, H31319, H31411, H31412, H31413, H31419, H3560, H3561, H3562, H3563, H35731, H35732, H35733, H35739, H4310, H4311, H4312, H4313, H44811, H44812, H44813, H44819, H47021, H47022, H47023, H47029, H59111, H59112, H59113, H59119, H59121, H59122, H59123, H59129, H59311, H59312, H59313, H59319, H59321, H59322, H59323, H59329, H9521, H9522, H9541, H9542, I312, I97410, I97411, I97418, I9742, I97610, I97611, I97618, I97620, J9561, J9562, J95830, J95831, L7601, L7602, L7621, L7622, M2500, M25011, M25012, M25019, M25021, M25022, M25029, M25031, M25032, M25039, M25041, M25042, M25049, M25051, M25052, M25059, M25061, M25062, M25069, M25071, M25072, M25073, M25074, M25075, M25076, M2508, M96810, M96811, M96830, M96831, N421, N857, N897, N920, N923, N930, N938, N939, N9961, N9962, N99820, N99821, R040, R041, R042, R0489, R049, R233, R310, R319, R58, T792XXA

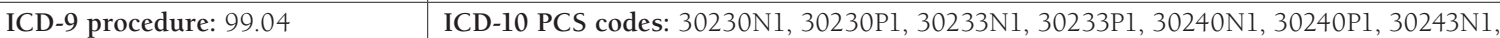
30243P1, 30250N1, 30250P1, 30253N1, 30253P1, 30260N1, 30260P1, 30263N1, 30263Pl

Cllinically relevant nonmajor bleeding
ICD-9-CM codes: $456.0 x, 456.20$, $530.82,531.0 x, 531.2 x, 531.4 x$, $531.6 x, 532.0 x, 532.2 x, 532.4 x$, $532.6 x, 533.0 x, 533.2 x, 533.4 x$, $533.6 x, 534.0 x, 534.2 x, 534.4 x$, $534.6 x, 535.01,535.11,535.21$, 535.31, 535.41, 535.51, 535.61, 537.83, 562.02, 562.03, 562.12, 562.13, 569.3, 569.85, 578.x

ICD-9-PCS codes: 44.43

ICD-9-Diagnosis: 285.1, 372.72, $374.81,596.7 x, 599.7 x, 602.1 x$ $620.1,621.4,626.2,626.5,626.7$,

Other major bleeding $626.8,626.9,782.7,784.7,784.8$, $786.3 x, 958.2$

ICD-9 procedure: 99.04

ICD-10-CM codes: I8501, I8511, K2211, K226, K250, K252, K254, K256, K260, K262, K264, K266, K270, K272, K274, K276, K280, K282, K284, K286, K2901, K2921, K2931, K2941, K2951, K2961, K2971, K2981, K2991, K31811, K3182, K5521, K5701, K5711, K5713, K5721, K5731, K5733, K5741, K5751, K5753, K5781, K5791, K5793, K625, K6381, K920, K921, K922, K9161, K9162, K91840, K91841

\section{ICD-10-PCS codes: no codes}

ICD-10-CM codes: D62, E89810, E89811, H0289, H1130, H1131, H1132, H1133, J9561, J9562, J95830, J95831, L7601, L7602, L7621, L7622, M96810, M96811, M96830, M96831, N421, N857, N897, N920, N923, N930, N938, N939, N9961, N9962, N99820, N99821, R040, R041, R042, R0489, R049, R233, R310, R319, R58, T792XXA

ICD-10-PCS codes: 30230N1, 30230P1, 30233N1, 30233P1, 30240N1, 30240P1, 30243N1, 30243Pl, 30250Nl, 30250Pl, 30253N1, 30253Pl, 30260Nl, 30260Pl, 30263N1, 30263Pl

Recurrent VTEa

PE: 415.11, 415.13, 415.19

DVT: 451.11, 451.19, 451.2, 451.81,

VTE 451.83, 451.84, 451.9, 453.1, 453.2, $453.4,453.82,453.83,453.84$ $453.85,453.86,453.87,453.89,453.9$
PE: I2692, I2699

DVT: I801, I802, I803, I809, I821, I82210, I82220, I82290, I8240, I8241, I8242, I8243, I8244, I8249, I824Y, I824Z, I8260, I8262, I82A1, I82B1, I82C1, I82890, I8290

aThe same codes were used to identify VTE patients for study inclusion.

DVT = deep vein thrombosis; ICD-9-CM=International Classification of Diseases, Ninth Revision, Clinical Modification; ICD-10-CM=International Classification of Diseases, Tenth Revision, Clinical Modification; PE= pulmonary embolism; VTE=venous thromboembolism. 
Comparative Clinical and Economic Outcomes Associated with Warfarin Versus Apixaban in the Treatment of Patients with Venous Thromboembolism in a Large U.S. Commercial Claims Database

APPENDIX B Descriptive Baseline Characteristics Among VTE Patients Prescribed Warfarin Versus Apixaban

\begin{tabular}{|c|c|c|c|c|c|c|c|c|c|c|}
\hline & \multicolumn{5}{|c|}{ Prematched } & \multicolumn{5}{|c|}{ Postmatched } \\
\hline & \multicolumn{2}{|c|}{$\begin{array}{l}\text { Apixaban Cohort } \\
\text { (Reference) }\end{array}$} & \multicolumn{2}{|c|}{ Warfarin Cohort } & \multirow[t]{2}{*}{ STD $^{a}$} & \multicolumn{2}{|c|}{$\begin{array}{l}\text { Apixaban Cohort } \\
\text { (Reference) }\end{array}$} & \multicolumn{2}{|c|}{ Warfarin Cohort } & $\mathrm{STD}^{\mathrm{a}}$ \\
\hline Sample size, $\mathrm{n}$ & \multicolumn{2}{|c|}{11,772} & \multicolumn{2}{|c|}{13,421} & & \multicolumn{2}{|c|}{8,858} & \multicolumn{2}{|c|}{8,858} & \\
\hline Age, mean (SD) & 52.7 & $(12.7)$ & 52.4 & $(13.1)$ & 1.89 & 52.6 & $(12.9)$ & 52.5 & (12.9) & 0.84 \\
\hline \multicolumn{11}{|l|}{ Age, ${ }^{b}$ years, $n(\%)$} \\
\hline $18-54$ & 5,956 & $(50.6)$ & 6,946 & $(51.8)$ & 2.33 & 4,527 & $(51.1)$ & 4,538 & $(51.2)$ & 0.25 \\
\hline $55-64$ & 4,252 & $(36.1)$ & 4,698 & $(35.0)$ & 2.34 & 3,162 & $(35.7)$ & 3,157 & (35.6) & 0.12 \\
\hline $65-74$ & 1,144 & $(9.7)$ & 1,214 & $(9.0)$ & 2.30 & 815 & $(9.2)$ & 830 & (9.4) & 0.58 \\
\hline $75-79$ & 155 & $(1.3)$ & 206 & $(1.5)$ & 1.84 & 130 & $(1.5)$ & 120 & $(1.4)$ & 0.96 \\
\hline$\geq 80$ & 265 & $(2.3)$ & 357 & $(2.7)$ & 2.64 & 224 & $(2.5)$ & 213 & $(2.4)$ & 0.80 \\
\hline \multicolumn{11}{|l|}{ Gender, ${ }^{b}$ n (\%) } \\
\hline Male & 6,554 & $(55.7)$ & 7,214 & $(53.8)$ & 3.85 & 4,907 & $(55.4)$ & 4,910 & $(55.4)$ & 0.07 \\
\hline Female & 5,218 & $(44.3)$ & 6,207 & $(46.2)$ & 3.87 & 3,951 & $(44.6)$ & 3,948 & $(44.6)$ & 0.07 \\
\hline \multicolumn{11}{|l|}{ Geographic region, ${ }^{b}$ n (\%) } \\
\hline Northeast & 2,281 & $(19.4)$ & 2,713 & $(20.2)$ & 2.11 & 1,993 & $(22.5)$ & 1,918 & $(21.7)$ & 2.04 \\
\hline North Central & 2,756 & $(23.4)$ & 4,348 & $(32.4)$ & 20.12 & 2,631 & $(29.7)$ & 2,643 & $(29.8)$ & 0.30 \\
\hline South & 5,216 & $(44.3)$ & 3,194 & $(23.8)$ & 44.32 & 2,732 & $(30.8)$ & 2,815 & $(31.8)$ & 2.02 \\
\hline West & 1,427 & $(12.1)$ & 2,990 & $(22.3)$ & 27.16 & 1,413 & $(16.0)$ & 1,401 & $(15.8)$ & 0.37 \\
\hline Other & 92 & $(0.8)$ & 176 & $(1.3)$ & 5.21 & 89 & $(1.0)$ & 81 & $(0.9)$ & 0.93 \\
\hline \multicolumn{11}{|l|}{ Plan type, n (\%) } \\
\hline Commercial & 5,437 & $(46.2)$ & 7,165 & $(53.4)$ & 14.43 & 4,014 & $(45.3)$ & 4,881 & $(55.1)$ & 19.67 \\
\hline Medicaid & 256 & $(2.2)$ & 1,408 & $(10.5)$ & 34.65 & 217 & $(2.4)$ & 659 & $(7.4)$ & 23.17 \\
\hline Medicare Advantage & 174 & $(1.5)$ & 337 & $(2.5)$ & 7.39 & 166 & (1.9) & 188 & $(2.1)$ & 1.77 \\
\hline Self-insured & 3,168 & $(26.9)$ & 3,774 & $(28.1)$ & 2.71 & 2,406 & $(27.2)$ & 2,642 & $(29.8)$ & 5.90 \\
\hline Medicare Supplemental & 11 & $(0.1)$ & 108 & $(0.8)$ & 10.65 & 8 & $(0.1)$ & 69 & $(0.8)$ & 10.48 \\
\hline Other/missing & 2,816 & $(23.9)$ & 767 & $(5.7)$ & 53.01 & 2,114 & $(23.9)$ & 510 & $(5.8)$ & 52.72 \\
\hline \multicolumn{11}{|l|}{ Index year, ${ }^{\mathrm{c}} \mathrm{n}(\%)$} \\
\hline 2014 & 158 & (1.3) & 2,094 & $(15.6)$ & 52.97 & 127 & $(1.4)$ & 1,407 & $(15.9)$ & 53.16 \\
\hline 2015 & 1,667 & $(14.2)$ & 5,472 & $(40.8)$ & 62.46 & 1,240 & $(14.0)$ & 3,635 & $(41.0)$ & 63.52 \\
\hline 2016 & 3,419 & $(29.0)$ & 3,443 & $(25.7)$ & 7.61 & 2,614 & $(29.5)$ & 2,207 & $(24.9)$ & 10.34 \\
\hline 2017 & 4,263 & $(36.2)$ & 1,885 & $(14.0)$ & 52.86 & 3,187 & $(36.0)$ & 1,264 & $(14.3)$ & 51.69 \\
\hline 2018 & 2,265 & $(19.2)$ & 527 & (3.9) & 49.28 & 1,690 & $(19.1)$ & 345 & (3.9) & 49.03 \\
\hline Type of index VTE encounter, ${ }^{b} \mathrm{n}(\%)$ & & & & & & & & & & \\
\hline Inpatient & 4,568 & $(38.8)$ & 7,494 & $(55.8)$ & 34.61 & 4,017 & $(45.3)$ & 4,113 & $(46.4)$ & 2.17 \\
\hline Outpatient & 7,204 & $(61.2)$ & 5,927 & $(44.2)$ & 34.61 & 4,841 & $(54.7)$ & 4,745 & $(53.6)$ & 2.17 \\
\hline ER & 3,613 & $(30.7)$ & 3,141 & $(23.4)$ & 16.46 & 2,410 & $(27.2)$ & 2,492 & $(28.1)$ & 2.07 \\
\hline Index VTE diagnosis, ${ }^{\mathrm{b}} \mathrm{n}(\%)$ & & & & & & & & & & \\
\hline DVT only & 6,455 & $(54.8)$ & 7,443 & $(55.5)$ & 1.26 & 4,947 & $(55.9)$ & 4,879 & $(55.1)$ & 1.54 \\
\hline PE with DVT & 1,874 & (15.9) & 2,126 & (15.8) & 0.23 & 1,457 & $(16.4)$ & 1,338 & $(15.1)$ & 3.69 \\
\hline PE without DVT & 3,443 & $(29.3)$ & 3,852 & $(28.7)$ & 1.20 & 2,454 & $(27.7)$ & 2,641 & $(29.8)$ & 4.66 \\
\hline Position of index VTE diagnosis, ${ }^{\mathrm{b}} \mathrm{n}$ & & & & & & & & & & \\
\hline Primary & 10,399 & $(88.3)$ & 11,716 & $(87.3)$ & 3.18 & 7,835 & $(88.5)$ & 7,841 & $(88.5)$ & 0.21 \\
\hline Secondary & 1,373 & $(11.7)$ & 1,705 & $(12.7)$ & 3.18 & 1,023 & $(11.5)$ & 1,017 & $(11.5)$ & 0.21 \\
\hline Index VTE etiology, ${ }^{\mathrm{b}} \mathrm{n}(\%)$ & & & & & & & & & & \\
\hline Provoked & 3,545 & $(30.1)$ & 4,335 & $(32.3)$ & 4.73 & 2,709 & $(30.6)$ & 2,741 & $(30.9)$ & 0.78 \\
\hline Unprovoked & 8,227 & $(69.9)$ & 9,086 & $(67.7)$ & 4.73 & 6,149 & $(69.4)$ & 6,117 & $(69.1)$ & 0.78 \\
\hline $\begin{array}{l}\text { Deyo-Charlson Comorbidity Index, } \\
\text { mean (SD) }\end{array}$ & 0.8 & (1.4) & 1.0 & (1.6) & 13.55 & 0.8 & $(1.5)$ & 0.8 & $(1.5)$ & 0.93 \\
\hline Baseline comorbidity, ${ }^{\mathrm{b}} \mathrm{n}(\%)$ & & & & & & & & & & \\
\hline AIDS & 57 & $(0.5)$ & 77 & $(0.6)$ & 1.23 & 44 & $(0.5)$ & 41 & $(0.5)$ & 0.49 \\
\hline Alcohol abuse & 393 & $(3.3)$ & 645 & $(4.8)$ & 7.43 & 318 & $(3.6)$ & 343 & $(3.9)$ & 1.49 \\
\hline Anemia & 1,720 & $(14.6)$ & 2,715 & $(20.2)$ & 14.83 & 1,417 & $(16.0)$ & 1,422 & $(16.1)$ & 0.15 \\
\hline Central venous catheter & 721 & $(6.1)$ & 1,253 & $(9.3)$ & 12.05 & 628 & $(7.1)$ & 633 & $(7.1)$ & 0.22 \\
\hline Cerebrovascular disease & 547 & $(4.6)$ & 979 & $(7.3)$ & 11.19 & 461 & $(5.2)$ & 456 & $(5.1)$ & 0.25 \\
\hline
\end{tabular}


Comparative Clinical and Economic Outcomes Associated with Warfarin Versus Apixaban in the Treatment of Patients with Venous Thromboembolism in a Large U.S. Commercial Claims Database

APPENDIX B Descriptive Baseline Characteristics Among VTE Patients Prescribed Warfarin Versus Apixaban (continued)

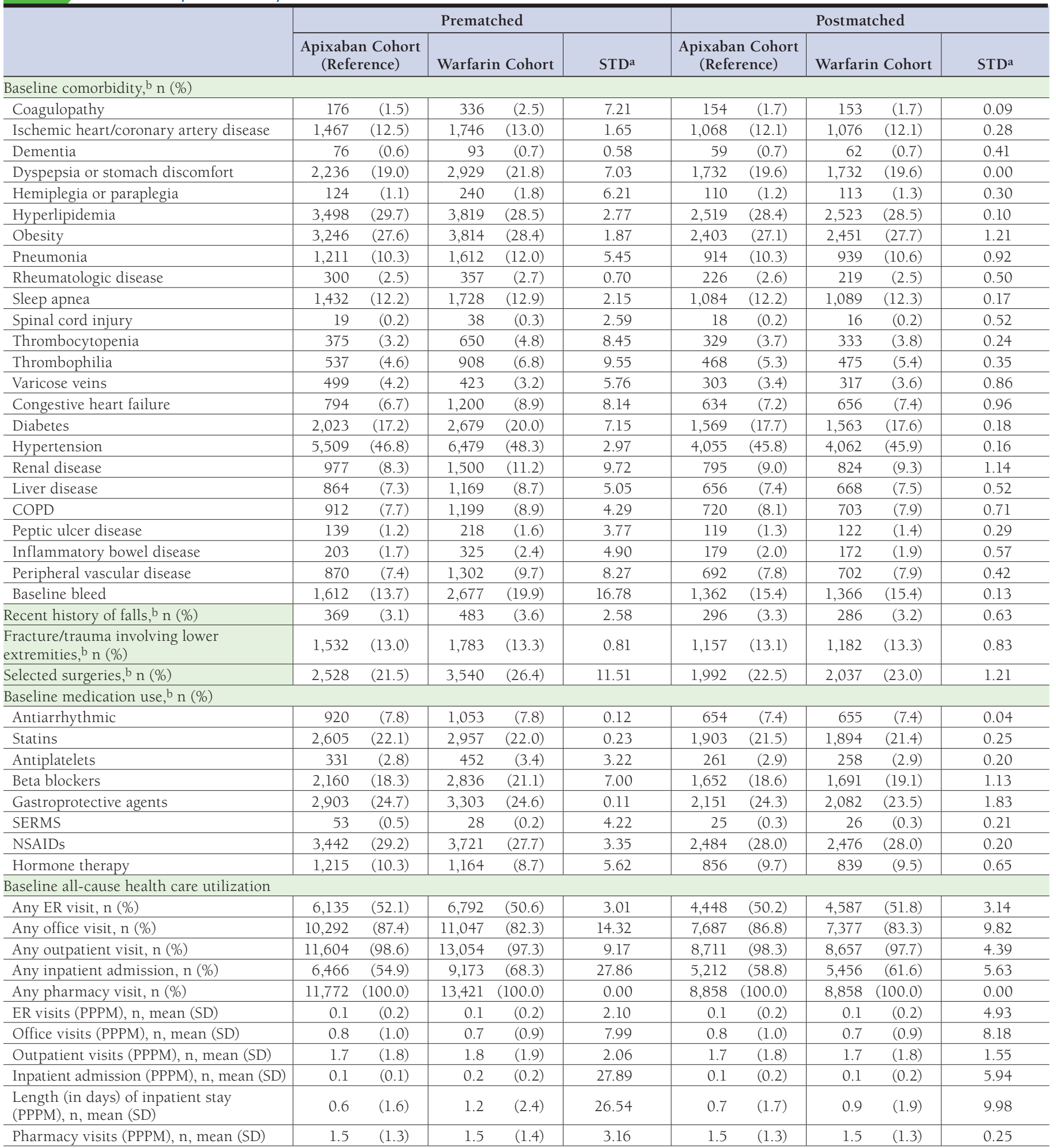


Comparative Clinical and Economic Outcomes Associated with Warfarin Versus Apixaban in the Treatment of Patients with Venous Thromboembolism in a Large U.S. Commercial Claims Database

\begin{tabular}{|c|c|c|c|c|c|c|}
\hline \multirow[t]{3}{*}{ APPENDIX B } & seline Chara & eristics Amon & $E P$ & s Prescribed & arfarin Versu & aban \\
\hline & \multicolumn{3}{|c|}{ Prematched } & \multicolumn{3}{|c|}{ Postmatched } \\
\hline & $\begin{array}{l}\text { Apixaban Cohort } \\
\text { (Reference) }\end{array}$ & Warfarin Cohort & STD $^{\mathrm{a}}$ & $\begin{array}{l}\text { Apixaban Cohort } \\
\text { (Reference) }\end{array}$ & Warfarin Cohort & STD $^{a}$ \\
\hline \multicolumn{7}{|c|}{ Baseline all-cause health care costs (PPPM), mean (SD) } \\
\hline ER visit costs & $95 \quad(241)$ & $(208)$ & 1.26 & $(196)$ & $(183)$ & 1.53 \\
\hline Office visit costs & $(111)$ & (111) & 2.71 & $(115)$ & $(107)$ & 4.61 \\
\hline Other outpatient costs & $796 \quad(2,002)$ & $795 \quad(1,918)$ & 0.02 & $784 \quad(2,170)$ & $777 \quad(1,813)$ & 0.34 \\
\hline Total outpatient costs (ER+office + other $)$ & $982(2,068)$ & $975 \quad(1,990)$ & 0.31 & $963(2,225)$ & $954(1,875)$ & 0.43 \\
\hline Inpatient stay costs & $2,527 \quad(5,818)$ & $4,072 \quad(8,911)$ & 20.53 & $2,805 \quad(6,267)$ & $3,198 \quad(7,156)$ & 5.83 \\
\hline Total medical costs (outpatient + inpatient) & $3,509 \quad(6,301)$ & $5,048 \quad(9,311)$ & 19.35 & $3,768 \quad(6,790)$ & $4,152 \quad(7,560)$ & 5.34 \\
\hline Pharmacy costs & $292 \quad(812)$ & $273 \quad(696)$ & 2.47 & $296 \quad(867)$ & $256 \quad(629)$ & 5.30 \\
\hline $\begin{array}{l}\text { Total health care costs } \\
\text { (outpatient }+ \text { inpatient }+ \text { pharmacy) }\end{array}$ & $3,801 \quad(6,404)$ & $5,321 \quad(9,379)$ & 18.92 & $4,065 \quad(6,896)$ & $4,408 \quad(7,632)$ & 4.72 \\
\hline \multicolumn{7}{|c|}{$\begin{array}{l}\text { aSTD: standardized difference }=100 \times \text { (actual STD). A standardized difference greater than } 10 \text { is considered significant. } \\
\text { bVariables that were included in } 1: 1 \text { propensity score matching between warfarin and apixaban patients. } \\
\text { 'Defined as the year in which patients had their index date. } \\
\text { AIDS=acquired immunodeficiency syndrome; COPD =chronic obstructive pulmonary disease; DVT= deep vein thrombosis; ER=emergency room; NSAIDS=nonsteroidal } \\
\text { anti-inflammatory drug; PE = pulmonary embolism; PPPM = per patient per month; SD = standard deviation; SERMS = selective estrogen receptor modulator; } \\
\text { STD= standardized difference; VTE= venous thromboembolism. }\end{array}$} \\
\hline
\end{tabular}

